\title{
Whole exome sequencing identifies multiple diagnoses in congenital glaucoma with systemic anomalies
}

Reis L.M., Tyler R.C., Weh E., Hendee K.E., Schilter K.F., Phillips J.A. III, Sequeira S., Schinzel A., Semina E.V. Whole exome sequencing identifies multiple diagnoses in congenital glaucoma with systemic anomalies. Clin Genet 2016: 90: 378-382. (C) John Wiley \& Sons A/S. Published by John Wiley \& Sons Ltd, 2016

The genetic basis of congenital glaucoma with systemic anomalies is largely unknown. Whole exome sequencing (WES) in 10 probands with congenital glaucoma and variable systemic anomalies identified pathogenic or likely pathogenic variants in three probands; in two of these, a combination of two Mendelian disorders was found to completely explain the patients' features whereas in the third case only the ocular findings could be explained by the genetic diagnosis. The molecular diagnosis for glaucoma included two cases with compound heterozygous or homozygous pathogenic alleles in CYP1B1 and one family with a dominant pathogenic variant in $\mathrm{FOXCl}$; the second genetic diagnosis for the additional systemic features included compound heterozygous mutations in NPHS1 in one family and a heterozygous $18 \mathrm{q} 23$ deletion in another pedigree. These findings show the power of WES in the analysis of complex conditions and emphasize the importance of CYP1B1 screening in patients with congenital glaucoma regardless of the presence/absence of other systemic anomalies.

\section{Conflict of interest}

The authors declare that they have no conflict of interest.

\author{
L. M. Reis ${ }^{a}$, R. C. Tylera, \\ E. Weh ${ }^{\mathrm{a}, \mathrm{b}}$, K. E. Hendee ${ }^{\mathrm{a}, \mathrm{b}}$, \\ K. F. Schiltera,b, \\ J. A. Phillips III' , S. Sequeirad, \\ A. Schinzel ${ }^{\mathbb{e}}$ and \\ E. V. Semina $a^{a, b, f}$
}

aDepartment of Pediatrics and Children's Research Institute, Medical College of Wisconsin and Children's Hospital of Wisconsin, Milwaukee, WI, USA, bDepartment of Cell Biology, Neurobiology and Anatomy, Medical College of Wisconsin, Milwaukee, WI, USA, 'Division of Medical Genetics and Genomic Medicine, Vanderbilt University Medical Center, Nashville, TN, USA,

dMetabolic Unit, Pediatric Department, Hospital de Dona Estefânia, CHLC, Lisbon, Portugal, e'Department of Genetics, Institute of Medical Genetics, Schwerzenbach, Switzerland, and fDepartment of Ophthalmology, Medical College of Wisconsin, Milwaukee, WI, USA

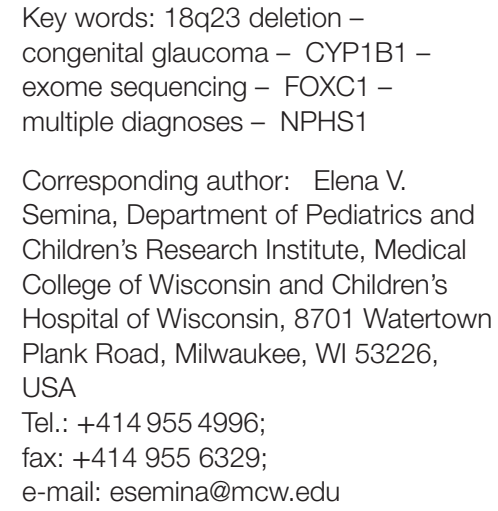

Key words: 18q23 deletion congenital glaucoma - CYP1B1 exome sequencing - FOXC1 multiple diagnoses - NPHS1

Corresponding author: Elena V. Semina, Department of Pediatrics and Children's Research Institute, Medical College of Wisconsin and Children's Hospital of Wisconsin, 8701 Watertown Plank Road, Milwaukee, WI 53226, USA

Tel.: +414955 4996;

fax: +414955 6329;

e-mail: esemina@mcw.edu

Received 24 March 2016, revised and accepted for publication 2 June 2016
Congenital glaucoma, also referred to as infantile, is characterized by onset in newborns or diagnosed in the first 2 years of life and may be classified as primary (PCG) in the absence of visible structural ocular anomalies or developmental when associated with anterior segment dysgenesis (1). CYP1B1 is the 
most common genetic cause of isolated congenital glaucoma, typically PCG, with mutations in LTBP2 occasionally reported as well $(1,2)$. Numerous genes have been identified in specific ocular syndromes involving congenital/infantile glaucoma in some cases including FOXCl and PITX2 (Axenfeld-Rieger syndrome), OCRL1 (Lowe syndrome), B3GLCT (Peters Plus syndrome), BMP4 (SHORT syndrome-like), TKS4 (Frank-ter Haar syndrome), CREBBP (Rubinstein-Taybi syndrome), POMT1, POMT2, and POMGNT1 [Walker Warburg and muscle-eye-brain (MEB) disease], $S B F 2$ (Charcot-Marie-Tooth Disease type 4B2), GNAQ (Sturge-Weber syndrome), RPS19 (Diamond Blackfan anemia), CANT1 (Desbuquois Dysplasia), BCOR (Oculofaciocardiodental syndrome), and GJA1 (Oculodentodigital dysplasia) (www.omim.org).

To evaluate the genetic causes of congenital glaucoma with systemic anomalies, we performed whole exome sequencing (WES) in 10 probands. This human study was approved by the Institutional Review Board of the Children's Hospital of Wisconsin with written informed consent obtained from every subject and/or their legal representative including consent for publication of photographs when applicable. The affected phenotypes involved congenital glaucoma with other eye anomalies, dysmorphic facial features, and developmental delay or cognitive impairment in the majority of patients $(8 / 10)$; additionally, short stature and/or structural birth defects affecting the heart, kidney, brain, or genitals were each present in about half of probands (Table S1, Supporting Information). WES was performed and analyzed as described previously (3) for variants in genes associated with congenital glaucoma (Table S2); other analyses included evaluation of variants in OMIM genes associated with pediatric conditions and/or present in ClinVar using the lists of genes/variants as developed by Golden Helix (Bozeman, MT, USA) and provided in their SNP and Variation Suite (SVS) software package. Allele frequency and reported pathogenicity were assessed using the ExAC browser (http://exac.broadinstitute.org/) and Human Gene Mutation Database (HGMD) (4). Variant pathogenicity was assessed using ACMG guidelines (5). Pathogenic variants were verified by Sanger sequencing in the probands and available family members. Exome sequencing data was also screened for copy number variation (CNV) using COPY NUMBER INFERENCE From EXome ReADS (CoNIFER) v0.2.2 software (http://conifer.sourceforge.net/); CNV analysis via Affymetrix Genome-Wide Human SNP Array 6.0 (Santa Clara, CA, USA) and TaqMan assays were also performed as previously described in one family (6). Pathogenic variants were submitted to the relevant LOVD 3.0 shared installation databases (patient IDs 00054892, 00054893, and 00059222).

Pathogenic/likely pathogenic variants were identified in three probands (Table 1); in two of these, a combination of two Mendelian disorders was found to fully explain the patients' features while in the third only the ocular findings could be explained by the genetic diagnosis. Review of the exome data on the remaining seven patients for sequence and copy number variants did not identify any pathogenic/likely pathogenic variants to explain the congenital glaucoma or syndromic anomalies.

Patient 1 (Fig. 1a,b), with bilateral congenital glaucoma with anterior segment dysgenesis, dysmorphic facial features, nephrotic syndrome, mild-moderate developmental delay, short stature, and feeding difficulties requiring placement of a gastric tube, was found to have compound heterozygous pathogenic/likely pathogenic variants in two genes: CYP1B1 and NPHS1 (Table 1); both unaffected parents were heterozygous carriers for these variants. The $C Y P 1 B 1$ variants have both been reported previously in patients with PCG (2). Congenital nephrotic syndrome of the Finnish type is a recessive condition caused by disruption of NPHSI typically associated with onset in the first 3 months of life with end-stage renal disease occurring by $2-3$ years of age (7). The NPHS1 nonsense variant seen in Patient 1 was previously reported as pathogenic (7). The missense change is a novel variant within the extracellular topological domain that is predicted to be damaging and is not present in ExAC; of note several similar missense variants affecting codons of neighboring amino acids have been reported as pathogenic (4).

Patient 2 (Fig. 1c,d), with congenital glaucoma and bilateral Peters anomaly, dysmorphic facial features, short stature, feeding issues requiring a nasogastric tube, intracranial calcifications, nephrocalcinosis and nephrolithiasis, and mild developmental delay, was found to have a homozygous previously reported pathogenic variant in CYP1B1; both unaffected parents were heterozygotes. No other pathogenic/likely pathogenic variants were identified in the whole exome data to explain the systemic features noted in this patient.

Patient 3, with congenital glaucoma, bilateral microphthalmia, iris anomalies, dysmorphic facial features, bilateral club foot (pes equinovarus), mild cognitive impairment (IQ about 60), congenital deafness, and a family history of variable non-congenital glaucoma, pupil anomalies, hearing loss and club foot in the father and paternal relatives (Fig. 1e), was found to have a pathogenic frameshift variant in $\mathrm{FOXCl}$ inherited from the father along with a de novo $4.8 \mathrm{Mb}$ terminal deletion of chromosome 18q23; Affymetrix microarray and TaqMan analysis confirmed the deletion on chromosome 18 from 73226420 to 78015057 in the proband and normal copy number in the parents and siblings (Fig. S1). While the FOXCl variant is novel, loss of function variants in $\mathrm{FOXCl}$ are a well-established cause of Axenfeld-Rieger syndrome type 3, associated with developmental glaucoma (sometimes congenital), iris anomalies, and dysmorphic facial features, with hearing impairment or heart defects in some cases (8). Distal 18q deletions are typically associated with cognitive impairment, craniofacial anomalies, hearing impairment, foot deformities including club foot, and short stature (9); microcornea has been reported in several patients (10). Thus, the congenital glaucoma and iris anomalies seen in the proband are likely due to the FOXCl mutation while the cognitive impairment and bilateral club foot 
Reis et al.

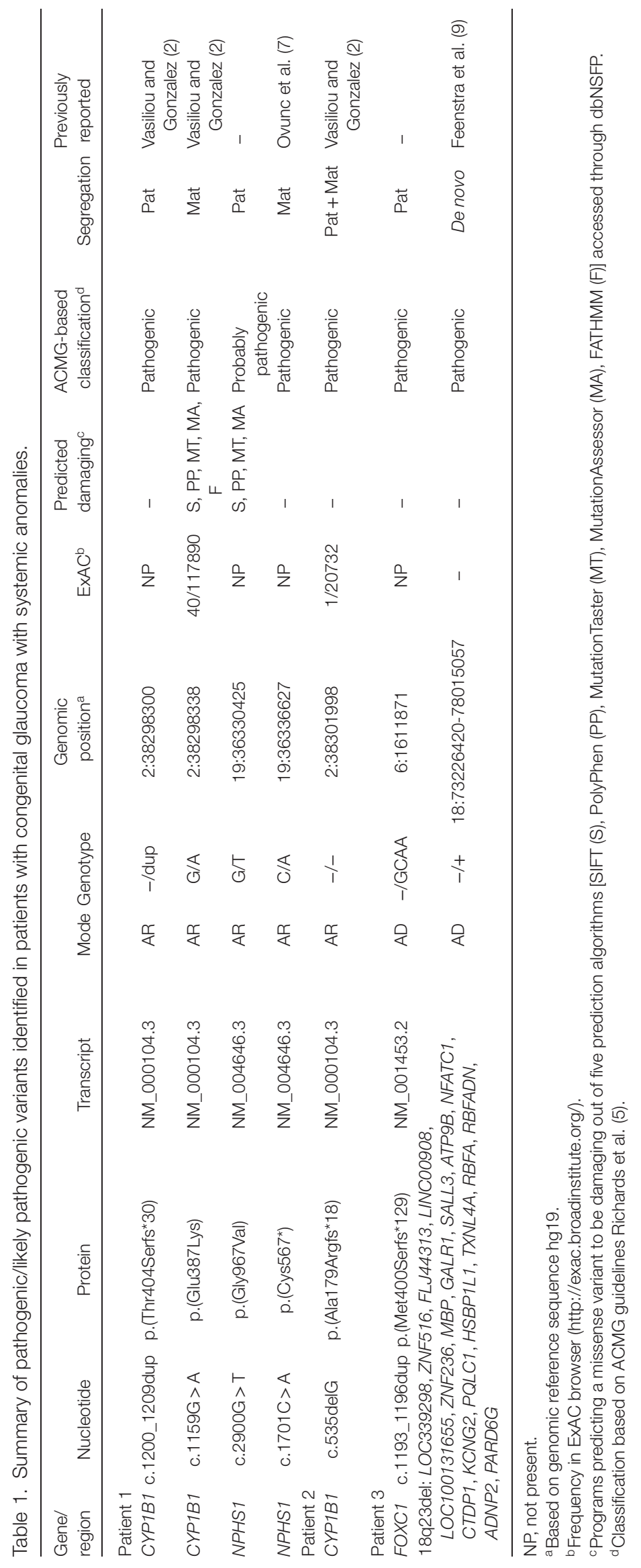



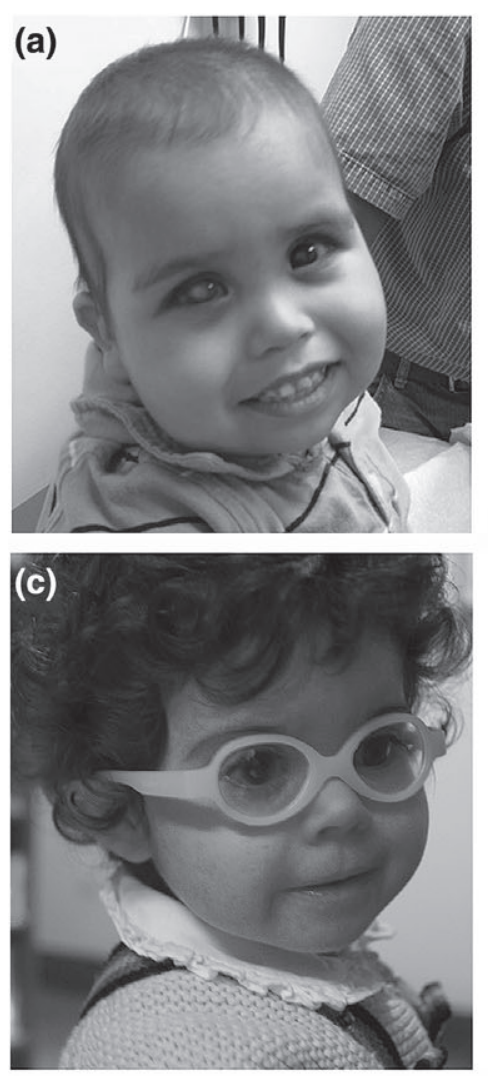

(b)

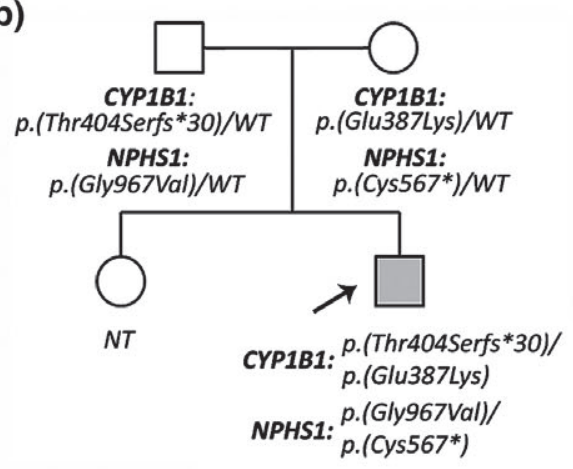

(d)

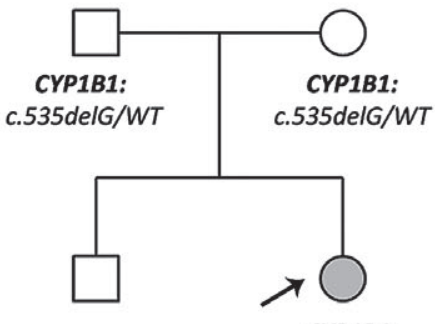

CYP1B1:

c.535delG/c.535delG

(e)

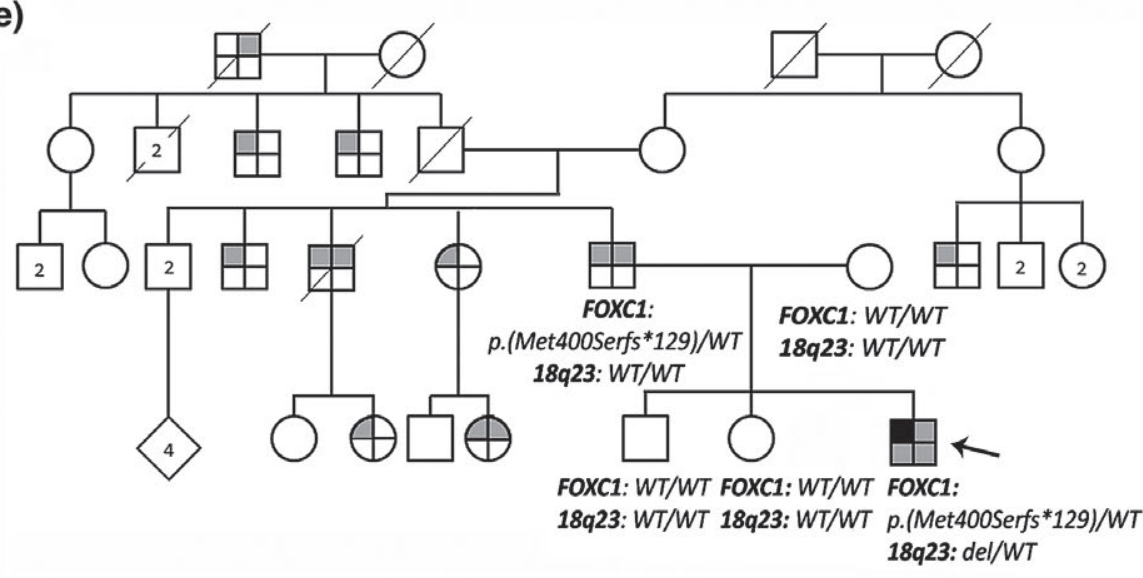

7 at least one of three: iris anomalies, non-congenital glaucoma, hearing loss

7 microphthalmia and dysmorphic features

Fig. 1. (a) Photograph of Patient 1 at 23 months of age with scaphocephaly, thin upper lip, mild mid-face hypoplasia, and a low-set ear on one side. (b) Pedigree of Patient 1 (marked with black arrow) with genotype information. (c) Photograph of Patient 2 at $\sim 20$ months of age. The patient demonstrated low-set posteriorly rotated ears, thin upper lip, maxillary hypoplasia, and deep set eyes. (d) Pedigree of Patient 2 (marked with black arrow) with genotype information. (e) Pedigree of Patient 3 (marked with black arrow) with genotype information.

fit better with the $18 \mathrm{q} 23$ deletion, although in this case club foot is also observed in the father and other paternal relatives. Because FOXCl deficiency and 18q deletions are both linked with dysmorphic facial features (midface hypoplasia, hypertelorism), hearing impairment, and ocular defects, the severe ocular and hearing features seen in Patient 3, congenital deafness and microphthalmia, are probably a result of the combined effect of the two genetic conditions.

WES was able to establish a genetic diagnosis in $30 \%$ (3/10) of probands affected with congenital glaucoma with systemic anomalies. Because these patients did not have features which fit with known glaucoma syndromes, it is not surprising that few of them had mutations 


\section{Reis et al.}

in the known genes. Our rate of diagnosis $(30 \%)$ is similar to the $25 \%$ success rate in other published exome analysis studies in cohorts of pediatric patients with an 'undiagnosed disease' (11). While blended phenotypes resulting from two single gene disorders were reported in the previous exome study [23/504 (4.6\%) of explained cases] (11), multiple diagnoses were identified in $67 \%$ of explained cases in our cohort, suggesting that congenital glaucoma with systemic anomalies that does not fit any specific syndrome may be more likely to be explained by two molecular diagnoses; further analysis is needed because it is possible that this increased rate is the result of the small sample size. The co-occurrence of two genetic conditions resulting from disruption of genes on different chromosomes would be expected to be a rare event. It is interesting to note that while the first patient's phenotype resulted from a combination of two distinct genetic conditions (congenital glaucoma and nephrotic syndrome), the third patient exhibited two genetic conditions with overlapping features and possible genetic interaction between the involved genes.

The identification of $C Y P 1 B 1$ mutations in two patients with congenital glaucoma with systemic anomalies highlights the importance of $C Y P 1 B 1$ screening in these cases despite its strong association with isolated PCG. Overall, these data suggest that WES is a useful tool for the diagnosis of congenital glaucoma with systemic anomalies.

\section{Supporting Information}

Additional supporting information may be found in the online version of this article at the publisher's web-site.

\section{Acknowledgements}

We are grateful to the families for their participation in this study. This project was supported by awards from National Institutes of Health/ National Eye Institute [grant number EY015518 to EVS]; Children's Research Institute Foundation at Children's Hospital of Wisconsin grant; Institute Core Facilities [grant number P30EY001931] and CTSA Grant from National Institutes of Health [grant number 1UL1RR031973].

\section{References}

1. Liu Y, Allingham RR. Molecular genetics in glaucoma. Exp Eye Res 2011: 93: 331-339.

2. Vasiliou V, Gonzalez FJ. Role of CYP1B1 in glaucoma. Annu Rev Pharmacol Toxicol 2008: 48: 333-358.

3. Deml B, Reis LM, Lemyre E, Clark RD, Kariminejad A, Semina EV. Novel mutations in PAX6, OTX2 and NDP in anophthalmia, microphthalmia and coloboma. Eur J Hum Genet 2016: 24: 535-541.

4. Stenson PD, Mort M, Ball EV, Shaw K, Phillips A, Cooper DN. The Human Gene Mutation Database: building a comprehensive mutation repository for clinical and molecular genetics, diagnostic testing and personalized genomic medicine. Hum Genet 2014: 133 (1): 1-9.

5. Richards S, Aziz N, Bale S et al. Standards and guidelines for the interpretation of sequence variants: a joint consensus recommendation of the American College of Medical Genetics and Genomics and the Association for Molecular Pathology. Genet Med 2015: 17: $405-424$.

6. Schilter KF, Reis LM, Schneider A et al. Whole-genome copy number variation analysis in anophthalmia and microphthalmia. Clin Genet 2013: 84 (5): 473-481.

7. Ovunc B, Ashraf S, Vega-Warner V et al. Mutation analysis of NPHS1 in a worldwide cohort of congenital nephrotic syndrome patients. Nephron Clin Pract 2012: 120 (3): c139-c146.

8. Reis LM, Tyler RC, Volkmann Kloss BA et al. PITX2 and FOXC1 spectrum of mutations in ocular syndromes. Eur J Hum Genet 2012: 20 (12): $1224-1233$.

9. Feenstra I, Vissers LE, Orsel M et al. Genotype-phenotype mapping of chromosome 18q deletions by high-resolution array CGH: an update of the phenotypic map. Am J Med Genet A 2007: 143A (16): 1858-1867.

10. Siddiqui SN, Levin AV, Rusinek M, Sutherland JE, Quinn AG. Ocular Manifestations of Chromosomal Abnormalities. In: Traboulsi EI, ed. Genetic Diseases of the Eye, 2nd edn. New York, NY: Oxford University Press, 2012: 224-225.

11. Yang Y, Muzny DM, Xia F et al. Molecular findings among patients referred for clinical whole-exome sequencing. JAMA 2014: 312 (18): $1870-1879$ 